Research Article

\title{
Internet and Mobile Phone Addiction Self-Control Mediate Physical Exercise and Subjective Well-Being in Young Adults Using IoT
}

\author{
Zhi-Chao Ding, Jun Yan $\mathbb{D}^{D}$, and Jian Fu \\ College of Physical Education, Yangzhou University, Yangzhou, Jiangsu 225009, China \\ Correspondence should be addressed to Jun Yan; 007587@yzu.edu.cn
}

Received 15 March 2021; Revised 24 April 2021; Accepted 8 May 2021; Published 18 May 2021

Academic Editor: Fazlullah Khan

Copyright ( 2021 Zhi-Chao Ding et al. This is an open access article distributed under the Creative Commons Attribution License, which permits unrestricted use, distribution, and reproduction in any medium, provided the original work is properly cited.

\begin{abstract}
Independent Internet surfing and persistently addictive smartphone use have become a significant public health problem. This addictive behavior is more prevalent in young people, who can engage in long-term secondary inopportune outgrowths. The main aim of this study is to see whether self-control in Internet usage (SCIU) and mobile phone addiction (MPA) will mediate the relationship between physical fitness and subjective well-being (SWB) in a group of Chinese adults using IoT. 1801 university students completed the proposed standard scale grading assignments. The results of our analysis showed that SCIU directly mediated the relationship between physical exertion and SWB. MPA mediated the interaction between physical exercise and SWB in reverse. The results also showed that the multiple SCIU-MPA serial frameworks between physical exercise and SWB were statistically significant. This result indicates that SCIU and MPA's relationships may be unique mediators in the relationship between physical exercise and SWB. Understanding the underlying interactions may have therapeutic significance for chronic, neurobiological disorders in young people with MPA. These results may also be used to improve physical intervention programs to improve the well-being of young Chinese people.
\end{abstract}

\section{Introduction}

Subjective well-being (SWB) is a concept used in the emerging field of positive psychology to explain how a person can live properly, develop emotional power, and succeed in his way of life over time [1]. The SWB comprises two elements: perceptual and affective. The cognitive factor refers to how an individual considers and assesses the satisfaction of his or her life in broad terms and in light of a specific set of personal expectations for their quality of life. While the affective side mainly relates to the client's mental well-being, it also explores the degree of emotional intensity and the quality of positive personal memories of glad moments in their lives [1-4]. In recent studies, everyday physical exercise's intensity has correlated with the SWB's cognitive and affective components [5]. Physical exercise provides individuals with a range of physical health benefits, including better cognitive ability, reducing the risk of fatal diseases, reducing cardiac arrest caused by weight imbalance and better body shape, as well as enhanced immunity. It has been shown that physical practice is effective. Such health advantages will dramatically improve the client's self-confidence by positive outcomes [6]. In earlier studies, daily physical workouts' psychological advantages, for example, reducing the effects of clinical depression and fear in young adults, have also consistently benefited $[7,8]$. Studies have also found that human participants who exercise regularly invoke more positive outcomes and fewer negative results, contributing to greater life satisfaction than people who do less [9]. Together, these experiments' findings show that the multimodal effects of exercise represent an improvement in mental well-being and an increased ability to perform regularly.

Although the association between physical exercise and SWB is explicit, the understanding of the mediating mechanisms can contribute to a better therapeutic strategy 
to relay the positive effect of physical exercise on mental health. Recently, several studies have found that this association is mediated by self-controlled Internet use and mobile phone addiction in young adults [10]. With the rapidly evolving globalization era, mobile phones serve as the medium for efficient communications and provide most of our daily-life information in real time, which has rendered this system to be more user-friendly, time-saving, and powerful tools compared to laptops. In our modern e-world, mobile phones allow access to social networks. They can increase productivity, thereby adding convenience to daily life, such as better social networking, better purchasing capacity, and greater knowledge about product variability and quality; however, lifestyle changes can have adverse effects if they affect daily work.

Under technological advancements in the telecommunication world, smarter mobile phone functionality and instant one-click access to messages, e-mails, and most popular browsers have increased psychological instability. It makes the young adults unaware of the passage of time and persistently dependent on the Internet [11]. Indeed, poor planning skills, low response inhibition, and the tendency to feel lost without their mobile phones and becoming bored easily are the characteristics of college students' addiction [12, 13]. Psychologically, this phenomenon is also considered a manifestation of symptoms of lack of self-control over voluntary tasks. Self-control has been defined as an individual's mental capacity to alter, modify, change, or override their impulses, desires, and habitual responses. Self-control in Internet use (SCIU) means that one can engage in the appropriate emotions, cognitions, and behaviors to effectively achieve selfrestraint on the Internet's unhealthy use. Extensive research has shown that people with regular physical exercise can control their Internet use behaviors and have less urge to rely on their phones, suggesting positive physical exercise effects on mental health and thoughts. More importantly, people with high self-control power may experience an improved quality of life and well-being [14]. Based on the available scientific evidence, we drew the following hypotheses.

Firstly, the connection between physical activity and SWB will be mediated by self-regulation of Internet usage. Excessive cell phone use can have serious long-term consequences, like mobile device addiction (MPA) $[15,16]$. It should be taken into account. MPA has been related to depression, anxiety, and stress in young adults, with some small inconsistencies. Furthermore, studies have shown that excessive cell phone use is associated with lower well-being and secondary psychiatric disorder and that a high level of MPA harms healthy teenage life [17].

Secondly, the MPA propensity will serve as a mediator between physical activity and SWB components. SCIU and MPA can both act as mediators in the relationship between physical activity and SWB components. However, from a neurological perspective, SCIU and MPA effects can be closely related. Addictive habits, according to self-regulation theory, are mainly the product of deficiencies of self-regulatory willpower. This loss of self-control can be inextricably related to the widespread use of smartphones in our everyday lives.
Finally, this research investigated the two mediation effects on college students' well-being compared to previous related studies that concentrated on the single impact of physical activity or MPA on the subject's well-being. Physical activity that affects college students' well-being differs from MPA that affects the well-being of young adults, so it is important to look at these young adults' mediation outcomes. By understanding the mediation effect specific to college students, universities and faculty will develop more tailored methods and relational strategies to improve young people's subjective well-being.

\section{Methods}

2.1. Procedure and Participants. The study sample consisted of Chinese young adults (18-22 years of age range) representative of the Chinese population regarding age, gender, educational level, and socioeconomic status. All participants were registered members of an online survey panel and were invited to participate in the study via WeChat. Participants were informed that their information would not be disclosed to third party without their further consent. By activating a link in WeChat, participants were directed to a web page to fill in several self-reported standard scales in September 2020. One thousand eight hundred and one university students were surveyed from 6 universities in Jiangsu, China. The survey was completed anonymously and confidentially, and all questionnaires were collected based on the voluntary principle. After further screening, 42 samples were found invalid as they did not meet the age criterion. Meanwhile, the other 34 samples were excluded for poor physical health status, which did not allow the participants to exercise in daily life. The final sample size analyzed in this study was one thousand seven hundred and twenty-five (mean age: $19.560 \pm 0.946)$, including nine hundred and eighty-five females.

2.2. Measures and Instruments. The Physical Exercise Rating Scale-3 (PARS-3) was developed by Takao Hashimoto in Japan and revised by Deqing Liang [18]. It has high reliability (retest reliability is 0.82 ). The volume of physical exercise was examined from three aspects: frequency of physical exercise, duration of physical exercise, and intensity of physical exercise, and the volume of physical exercise was used to measure the degree of participation in physical exercise. The measurement items of physical exercise behavior were (1) the intensity of physical exercise each time; (2) the duration in one session when doing the intensity as mentioned earlier sports activities; and (3) the times for performing the above sports activities a month. The first and second items were scored on a scale of 1-5 points, and the third item was scored on a scale of $0-4$ points. The higher the score of PARS-3 was, the greater the volume of physical exercise was. This scale has acceptable reliability and validity by a previously published report [19]. The Cronbach's alpha in our study was 0.670 .

The Index of Well-being Scale-China Revised (IWBS-cr) was applied to assess the informants' level of SWB during the 
present time [20]. The scale includes two dimensions of subjective well-being: affection (eight items) and overall life satisfaction (one item). The options for life satisfaction ranged from 1 (extremely dissatisfied) to 7 (extremely satisfied), and the options for affection were rated by pairs of opposite feeling ranging from 1 (i.e., boring) to 7 (i.e., quite interesting). Total scores were calculated using the following formula: index $=1.1 \times$ (overall life satisfaction item score $)+1.0 \times$ (mean of eight general affect scores). Total scores ranged between 2.10 and 14.70, and higher scores indicated higher levels of SWB. Previous research has shown that the IWBS is psychometrically sound for use with Chinese adults. In this study, the Cronbach's alpha coefficient was 0.955 in our study. Internet use of Selfcontrol Scale for college students (IUSCS-cs) was administrated following the previously published protocol. The scale comprises three subscales-emotion control, cognitive control, and the behavior of Internet use. The internal consistency of the original scale was good in our study $(\alpha=0.883)$. The scale used to measure the self-control power for college students has good reliability and validity. Higher scores indicated higher levels of SCIU. The Mobile Phone Addiction Tendency scale (MPATS) based on Young's Internet addiction scale was adopted. The items examined perceptions of college students' withdrawal symptoms (six items), salience (four items), social comfort (three items), and mood changes (three items). All 16 items were rated on 5-point scales ranging from 0 (very inconsistent) to 4 (very consistent). Items were summed to create a composite score for each respondent, with higher scores indicating a greater perceived tendency of MPA. The MPATS had good internal consistency in our study $(a=0.933)$. Previous research has shown that the MPAS is a valid, psychometrically sound instrument to assess Chinese adults MPA.

\section{Statistical Analysis}

To analyze results, SPSS and PROCESS [81] used freely available computational macros for SPSS that incorporate mediation and moderation processing. A statistically relevant $p$ value of 0.05 was used. To assess the strength of interaction between variables, the Pearson correlation coefficient was calculated. Model 6 of the PROCESS template, which specified a chain mediator model, was used to evaluate SC and MPD's mediating function. Centered on 5,000 resamples, the macros presented a model of overall effects, direct and indirect effects, and bootstrap confidence intervals.

\section{Experimental Results}

In this section, we analyze and determine the proposed model's performance and efficacy and evaluate the application apprehending IoT for self-control in Internet usage and mobile phone addiction to strengthen the study. We use the traditional approach bias assessment to find means, standard deviations, and correlation matrices for the physical activity, SCIU, and MPAT variables.
4.1. Experimental Setup. We have set up an IoT sensing cluster with default configurations. The $\mathrm{C} / \mathrm{C}++$ network is used to build the related graphical model of vocal art expression and body noise. The message link model and control of the wireless sensing vocal music node are also built on the same configuration. The node transmission data simulation time is $100 \mathrm{~s}$, with the SNR being $3 \mathrm{~dB}$.

4.2. Common Method Bias (CMB) Tests. A significant possible cause of $\mathrm{CMB}$ is using the same research respondent, i.e., a common source for information on both the dependent and independent variable measures [21]. We first use the Harman single factor test, and the results are shown in Table 1. From Table 1, we can observe that the total fluctuation is $21 \%$, which is below $40 \%$.

In our situation, the CMB did not consider that the popular approach bias could often occur from respondents' propensity to respond consistently. Moreover, if higher associations exist with sample variables $(r>.90)$, the CMB remains constant. According to the correlation results, higher associations were not found between the comparison variables. These statistics indicate that a typical process distortion in the data did not create a significant concern.

4.3. Common Method Variance Analysis. This section assesses the proposed work's output using means, standard deviations, and variable correlation matrices. Table 2 shows the success results. Table 2 shows that the college's physical activity, SCIU, and MPAT are positively connected to the children's subjective well-being $(p=0.01)$. College students' physical activity and SCIU were positively associated with their SWB, while MPAT was inversely linked with SWB.

In the above model, the normalized variables also determined the regression equation frequency. Table 3 shows the effects of SCIU and MPA chain mediations between PE and SWB. Following the regression and the bootstrap method [22], the chain mediation effects of SCIU and MPAT were established between PE and SWB. The PE score is the independent variable, and the SWB score is the dependent variable, where the SCIU and MPAT values are the variables of mediation. Unstandardized regression coefficients are estimated to minimize type- 1 error induced by the data distribution. The significance of path coefficients and selfconfidence periods for total, direct, and indirect effects were calculated through regression and bootstrapping methods. If the $95 \%$ bias-corrected bootstrap confidence intervals do not include zero, the mediation effects are significant.

Figure 1 also demonstrates numerous serial mediations between PE and SWB data. In Figure 1, we can see the statistically important overall influence of PE on SWB and the immediate effect. The statistically significant influence based on the confidence interval is the PE function SCIU file, the PE file MPAT file, and the PE function SCIU file. Moreover, three indirect effects were statistically significant based on confidence intervals. The overall indirect effects are $0.018,0.008$ ( $\mathrm{a} 1$ or b1), and 0.009 ( $\mathrm{a} 2$ or b2), respectively. The share of the gross indirect influence and three branch-indirect effects was $41.86 \%, 18.60 \%, 20.93 \%$, and $2.33 \%$. 
TABLE 1: Common method bias-variance analysis results and deep learning.

\begin{tabular}{lccc}
\hline \multicolumn{3}{c}{ Initial eigenvalue } \\
Factor & DNN ACC (\%) & Cumulative (\%) & \% of variance \\
\hline 1 & 92.11 & 18.99 & 26.34 \\
2 & 88.56 & 17.94 & 11.89 \\
3 & 85.32 & 16.89 & 34.23 \\
4 & 86.43 & 15.84 & 22.34 \\
5 & 82.45 & 14.79 & 15.87 \\
\hline
\end{tabular}

TABLe 2: Variance analysis variables $(N=1786)$.

\begin{tabular}{lcccccc}
\hline Methods & $M$ & SD & PEV & SCIU & MPAT & SWB \\
\hline PEV & 16.86 & 18.038 & - & - & - & - \\
SCIU & 79.83 & 17.477 & 0.347 & - & - & - \\
MPAT & 38.63 & 12.854 & -0.445 & -0.315 & - & - \\
SWB & 6.521 & 2.757 & 0.279 & 0.252 & -0.281 & - \\
\hline
\end{tabular}

TABLE 3: Regression analysis of the mediation models between physical exercise and SWB $(N=1786)$.

\begin{tabular}{lcccc}
\hline Path & Effect & Boot SE & Boot LLCI & Boot ULCI \\
\hline Direct effect & & & & \\
a1 & 0.314 & 0.023 & 0.269 & 0.358 \\
a2 & -0.265 & 0.017 & -0.297 & -0.232 \\
a3 & -0.132 & 0.017 & -0.165 & -0.099 \\
b1 & 0.024 & 0.004 & 0.016 & 0.031 \\
b2 & -0.036 & 0.006 & -0.047 & -0.025 \\
Total & 0.025 & 0.004 & 0.017 & 0.033 \\
\hline Indirect effects & & & & \\
$\quad$ Indirect 1 & 0.007 & 0.001 & 0.005 & 0.01 \\
Indirect 2 & 0.01 & 0.002 & 0.007 & 0.013 \\
Indirect 3 & 0.002 & 0.0003 & 0.001 & 0.002 \\
Total & 0.019 & 0.002 & 0.014 & 0.023 \\
\hline Total effect & 0.044 & 0.005 & 0.022 & 0.043 \\
\hline
\end{tabular}

\section{Discussion}

The basic aim of this research was to test the multimodal effects on SWB components of physical activity. The findings demonstrated that the few SCIU and MPA serial mediations between physical exercise and SWB were statistically significant. The idea that good health will enhance selfawareness and daily physical activity (such as self-discipline of Internet users) has resonated widely. These results helped to illustrate how frequent or regular training would contribute to physical and mental health well-being. Studies have shown that both time and physical exercise affecting the entire body are key factors in achieving maximal benefits than any particular form of physical activity. Also, the overall everyday living of individuals was an important factor in maintaining persistent subjective welfare, as was the success of the physical exercise alone. According to our first hypothesis, the physical fitness of college students was closely associated with SWB. This effect allows individuals to sustain their healthier lifestyles, positive eating habits, a longer sense of bliss, and often a normal way to maintain their corporal functions and manage tension and anxiety throughout their day-to-day lives, thereby increasing their average lifespan.
Two possible pathways have explained the effects of physical exercise on subjective well-being. The first involves improving physical condition, including aerobic status, body strength, and functionality $[23,24]$. Secondly, psychological variables such as reduced anxiety and depression may lead to improved self-performance, self-regulation, attitudes, and mental well-being. Furthermore, people with mental disabilities could benefit from routine physical workouts to improve their happiness. New research shows that people exercise less before the COVID-19 pandemic, and then exercise more during the pandemic; however, no major mood changes were found compared to those inactive during the COVID-19 epidemic. Consequently, the findings showed that in most cases, there is a similar connection between physical activity involvement and SWB. Physical activity has directly and implicitly predicted SWB by SCIU following our speculation. The mental exercise of individuals that limit previously strong responses (such as behavioral trends, emotions, and motivation) is self-control. Many facets of life demand self-control, such as resisting impulsive behavior, managing tension, delaying gratification, filling up time schedules, and so on. In the minimal energy selfregulation model, Muraven and Baumeister explicitly claimed that all self-control behavior and the extent of this energy specifically defines the values of the control result. People with strong self-control capacities usually report good effects, such as greater ecological and behavioral adaptability, improved school and job performance, and more satisfaction.

In the present study, SWB was predicted by physical activity via MPA directly and indirectly. These results support several earlier works on physical workouts that could reduce the possibility of overusing cell phones. Intensive mobile telephone use will lead to addiction trends and low life satisfaction in other life-related areas. Recent studies show that physical exercise can moderate mental health problems and thus decrease the risk of addictive trends. Exercise by controlling central and autonomous nervous processes seems to reduce addiction via the Internet [25]. Thus, Internet and mobile addiction reduction exercise-based approaches can be recommended. The present serial study of various mediations found that SWB was indirectly affected by SCIU and MPA exercises. Different studies have shown that the dependence of young people on mobile phones has become a normal phenomenon. To limit the use of cell phones, individuals must utilize cognitive inhibition, i.e., an inhibitor of self-control. The longer cycle and greater duration of exercise will lead. They, in turn, can have less time to cope with problematic addiction habits such as uncontrolled use of cell telephones and, as a result, improve their self-control capability. Too many applications of mobile phones often lead to unstable lifestyles and sedentary behavior problems, and at the same time there is almost no opportunity for exercise, resulting in health disadvantages such as obesity or metabolism, lower back, neck and shoulder pain, blurred vision and depression influences. Overall, this research strongly shows a significant correlation between self-control of Internet usage and the addictive use of cell telephones. 


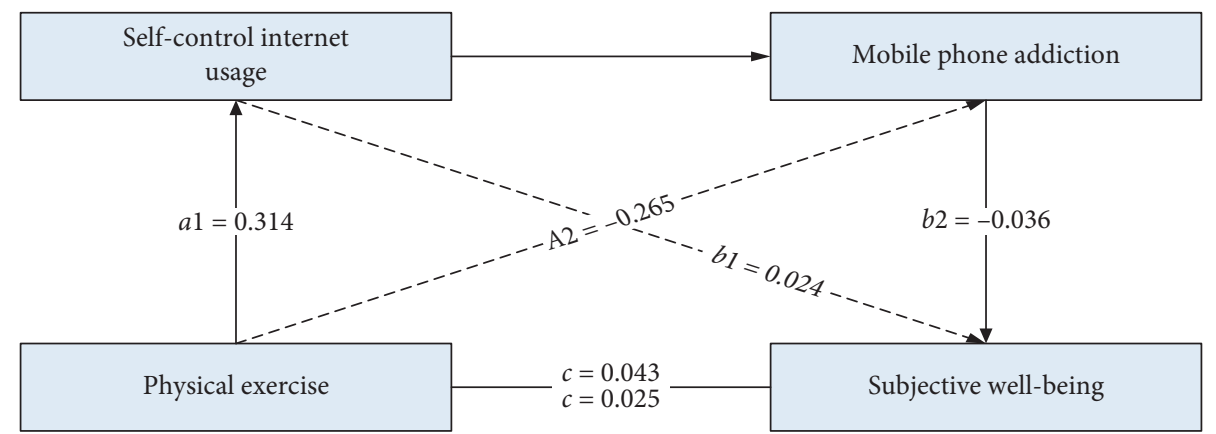

FIgURE 1: The serial multiple mediations of SCIU and MPAT between PE and SWB.

\section{Conclusions}

Physical practice impacts the automated usage of the Internet and the troublesome use of smartphones by students. Internet self-control and problem-sensitive mobile usage will mediate the link between exercise and well-being. This research has shown that physical fitness workers can benefit from more mental well-being by behavioral health and meeting the target of reducing cell phone dependence. Besides, intervention fitness activities may be used to support China's young adults' well-being. These strategic programs will increase their self-control ability throughout the Internet and at the same time reduce smartphone addiction. There are still some limitations to be acknowledged in this experiment. An assessment evaluated the amount of physical activity. Therefore, participants subjectively recorded amounts of physical fitness, which in certain circumstances may be misleading. In the future, more detailed measurements and in-depth surveys may determine the amount of physical activity and physical fitness that predicts the psychological well-being of young adults.

\section{Data Availability}

The datasets used and/or analyzed during the current study are available from the corresponding author on reasonable request.

\section{Conflicts of Interest}

The authors declare that they have no conflicts of interest.

\section{Acknowledgments}

This research was funded by the Humanities and Social Science Foundation of Yangzhou University (grant no. XJ2019-14).

\section{References}

[1] E. Diener, "Subjective well-being," Psychological Bulletin, vol. 95, no. 3, pp. 542-575, 1984.

[2] E. Diener, "Assessing subjective well-being: progress and opportunities," in Social Indicators Research Series: Assessing Well-Being: The Collected Works of Ed Diener, E. Diener, Ed., vol. 39, pp. 25-65, Springer, Berlin, Germany, 2009.
[3] E. Diener, S. Oishi, and R. E. Lucas, "Personality, culture, and subjective well-being: emotional and cognitive evaluations of life," Annual Review of Psychology, vol. 54, no. 1, pp. 403-425, 2003.

[4] S. Lyubomirsky, "Why are some people happier than others? The role of cognitive and motivational processes in wellbeing," American Psychologist, vol. 56, no. 3, pp. 239-249, 2001.

[5] H.-Y. An, W. Chen, C. W. Wang, H. F. Yang, W. T. Huang, and S. Y. Fan, "The relationships between physical activity and life satisfaction and happiness among young, middle-aged, and older adults," International Journal of Environmental Research and Public Health, vol. 17, no. 13, Article ID 4817, 2020.

[6] R. Brand, S. Timme, and S. Nosrat, "When pandemic hits: exercise frequency and subjective well-being during COVID19 pandemic," Frontiers in Psychology, vol. 11, Article ID 570567, 2020.

[7] S. Buecker, T. Simacek, B. Ingwersen, S. Terwiel, and B. A. Simonsmeier, "Physical activity and subjective wellbeing in healthy individuals: a meta-analytic review," Health Psychology Review, vol. 2020, 19 pages, 2020.

[8] A. García-Hermoso, I. Hormazábal-Aguayo, O. FernándezVergara, P. R. Olivares, and X. Oriol-Granado, "Physical activity, screen time and subjective well-being among children," International Journal of Clinical and Health Psychology, vol. 20, no. 2, pp. 126-134, 2020.

[9] M. Mutz, "Forced adaptations of sporting behaviours during the Covid-19 pandemic and their effects on subjective wellbeing," European Societies, vol. 23, no. 1, pp. S184-S198, 2020.

[10] J. González-Hernández, M. Gómez-López, J. A. Pérez-Turpin, A. J. Muñoz-Villena, and E. Andreu-Cabrera, "Perfectly active teenagers. When does physical exercise help psychological well-being in adolescents?" International Journal of Environmental Research and Public Health, vol. 16, no. 22, Article ID 4525, 2019.

[11] P. Luna, J. Guerrero, and J. Cejudo, "Improving adolescents' subjective well-being, trait emotional intelligence and social anxiety through a programme based on the sport education model," International Journal of Environmental Research and Public Health, vol. 16, no. 10, Article ID 1821, 2019.

[12] G. A. Panza, B. A. Taylor, P. D. Thompson, C. M. White, and L. S. Pescatello, "Physical activity intensity and subjective well-being in healthy adults," Journal of Health Psychology, vol. 24, no. 9, pp. 1257-1267, 2019.

[13] S. Pengpid and K. Peltzer, "Sedentary behaviour, physical activity and life satisfaction, happiness and perceived health status in university students from 24 countries," International 
Journal of Environmental Research and Public Health, vol. 16, no. 12, Article ID 2084, 2019.

[14] B. C. L. Chan, M. Luciano, and B. Lee, "Interaction of physical activity and personality in the subjective wellbeing of older adults in Hong Kong and the United Kingdom," Behavioral Sciences, vol. 8, no. 8, p. 71, 2018.

[15] M. A. Martinez-Gonzalez, "Physical activity and psychological well-being: edited by S J H Biddle, K R Fox, S H Boutcher. (Pp 240; \$29.99). Book News, 2000. ISBN 0-4152-3439-5," Journal of Epidemiology \& Community Health, vol. 57, no. 3, pp. 232-b, 2003.

[16] G. Raghuveer, J. Hartz, D. R. Lubans et al., "Cardiorespiratory Fitness in Youth: An Important Marker of Health: A Scientific Statement From the American Heart Association," Circulation, vol. 142, no. 7, pp. e101-e118, 2020.

[17] P. Galan-Lopez and F. Ries, "Motives for Exercising and Associations with Body Composition in Icelandic Adolescents," Sports, vol. 7, no. 6, p. 149, 2019.

[18] C. J. Lavie, R. Arena, D. L. Swift et al., "Exercise and the Cardiovascular System," Circulation Research, vol. 117, no. 2, pp. 207-219, 2015.

[19] J. R. Best, B. K. Chiu, C. Liang Hsu, L. S. Nagamatsu, and T. Liu-Ambrose, "Long-Term Effects of Resistance Exercise Training on Cognition and Brain Volume in Older Women: Results from a Randomized Controlled Trial," Journal of the International Neuropsychological Society, vol. 21, no. 10, pp. 745-756, 2015.

[20] S. Hong, J. Lee, J. Park et al., “Association between cardiorespiratory fitness and the prevalence of metabolic syndrome among Korean adults: a cross sectional study," BMC Public Health, vol. 14, p. 481, 2014.

[21] P. T. Nikolaïdis, "Physical fitness is inversely related with body mass index and body fat percentage in soccer players aged 1618 years," Medicinski Pregled, vol. 65, no. 11-12, pp. 470-475, 2012.

[22] S. Meeuwsen, G. W. Horgan, and M. Elia, "The relationship between BMI and percent body fat, measured by bioelectrical impedance, in a large adult sample is curvilinear and influenced by age and sex," Clinical Nutrition, vol. 29, no. 5, pp. 560-566, 2010.

[23] U. Ekelund, E. Poortvliet, A. Nilsson, A. Yngve, A. Holmberg, and M. Sjöström, "Physical activity in relation to aerobic fitness and body fat in 14- to 15-year-old boys and girls," European Journal of Applied Physiology, vol. 85, no. 3-4, pp. 195-201, 2001.

[24] J. Brailovskaia and J. Margraf, "Relationship between depression symptoms, physical activity, and addictive social media use," Cyberpsychology, Behavior, and Social Networking, vol. 23, no. 12, pp. 818-822, 2020.

[25] A. Lindegård, G. Wastensson, E. Hadzibajramovic, and A. Grimby-Ekman, "Longitudinal associations between cardiorespiratory fitness and stress-related exhaustion, depression, anxiety and sleep disturbances," BMC Public Health, vol. 19, no. 1, Article ID 1726, 2019. 